\title{
Serum lipid level is not associated with symptomatic intracerebral hemorrhage after intravenous thrombolysis for acute ischemic stroke
}

\author{
Ting-Chun Lin ${ }^{\text {Corresp.. }}{ }^{1}$, Yen-Kuang Lin ${ }^{2}$, Chin-I Chen ${ }^{3}$, Lung Chan ${ }^{4}$, Nai-Fang Chi ${ }^{4}$, Rey-Yue Yuan ${ }^{1}$, Jau- \\ Jiuan Sheu ${ }^{1}$, Chun-Ren Wei ${ }^{1}$, Jui-Ping Tsai ${ }^{1}$, Tu-Hsueh Yeh ${ }^{1}$ \\ 1 Department of Neurology, Taipei Medical University Hospital, Taipei Medical University, Taipei, Taiwan \\ 2 Research Center of Biostatistics, Taipei Medical University, Taipei, Taiwan \\ 3 Department of Neurology, Wan Fang Hospital, Taipei Medical University, Taipei, Taiwan \\ 4 Department of Neurology, Shuang Ho Hospital, Taipei Medical University, New Taipei City, Taiwan \\ Corresponding Author: Ting-Chun Lin \\ Email address: Lteikin@gmail.com
}

\section{Background}

This study assessed whether serum lipid levels are associated with the risk of symptomatic intracerebral hemorrhage $(\mathrm{sICH})$ and functional outcomes in patients with acute ischemic stroke after receiving intravenous thrombolysis.

\section{Methods}

We retrospectively analyzed consecutive ischemic stroke patients who were treated with intravenous tissue plasminogen activator between January 2007 and January 2017. Lipid levels on admission, including total cholesterol, low-density lipoprotein cholesterol (LDL-C), high-density lipoprotein cholesterol (HDL-C), and triglyceride levels, as well as potential predictors of SICH were tested using univariate and multivariate analyses.

\section{Results}

Of the 229 enrolled patients (100 women, aged $68 \pm 13$ years), 14 developed sICH and 103 (45\%) had favorable functional outcomes at 3 months. The patients with sICH more often had diabetes mellitus (71\% vs. $26 \%, \mathrm{P}=0.01$ ) and had more severe stroke (mean National Institutes of Health Stroke Scale [NIHSS] score of 16 vs. 13, P = 0.045). Regarding lipid subtype, total cholesterol, LDL-C, HDL-C, and triglyceride levels were not associated with $\mathrm{sICH}$ or functional outcomes. According to the results of multivariate analysis, the frequency of sICH was independently associated with diabetes mellitus (odds ratio $[\mathrm{OR}]=6.04 ; 95 \%$ confidence interval $[\mathrm{Cl}]=1.31-27.95 ; \mathrm{P}=0.02)$ and the NIHSS score $(\mathrm{OR}=1.12$; $95 \% \mathrm{Cl}=1.02-1.22 ; \mathrm{P}=0.01)$. A higher NIHSS score was independently associated with unfavorable functional outcomes $(\mathrm{OR}=0.86 ; 95 \% \mathrm{Cl}=0.81-0.91 ; \mathrm{P}<0.001)$.

\section{Conclusions}

Serum lipid levels on admission, including total cholesterol, LDL-C, HDL-C, and triglyceride levels, were not associated with $\mathrm{sICH}$ or 3-month functional outcomes after intravenous thrombolysis for acute ischemic stroke. 
1 Serum lipid level is not associated with symptomatic intracerebral hemorrhage after

2 intravenous thrombolysis for acute ischemic stroke

3

4 Ting-Chun Lin ${ }^{1}, \mathrm{MD}$; Yen-Kuang Lin ${ }^{2}, \mathrm{PhD}$; Chin-I Chen ${ }^{3}$, MD; Lung Chan ${ }^{4}$, MD; Nai-Fang

$5 \mathrm{Chi}^{4}, \mathrm{MD}$; Rey-Yue Yuan ${ }^{1}, \mathrm{MD}$; Jau-Jiuan Sheu ${ }^{1}, \mathrm{MD}$; Chun-Ren Wei ${ }^{1}$, MD; Jui-Ping Tsai ${ }^{1}$,

$6 \mathrm{MD}$; Tu-Hsueh $\mathrm{Yeh}^{1} \mathrm{MD}, \mathrm{PhD}$

7

$8{ }^{1}$ Department of Neurology, Taipei Medical University Hospital, Taipei, Taiwan

$9 \quad 2$ Research Center of Biostatistics, Taipei Medical University, Taipei, Taiwan

$10{ }^{3}$ Department of Neurology, Wan Fang Hospital, Taipei Medical University, Taipei, Taiwan

$11{ }^{4}$ Department of Neurology, Shuang Ho Hospital, Taipei Medical University, New Taipei City,

12 Taiwan

13

14

15 Corresponding author :

16 Ting-Chun, Lin. M.D.

17 Department of Neurology, Taipei Medical University Hospital

18 Address: No.252, Wu Hsing Street, Taipei City 110, Taiwan(R.O.C.)

19 TEL: +886-2-27372181 ext.8257

20 FAX: +886-2-27372758 
21 E-mail: Lteikin@gmail.com; 131004@h.tmu.edu.tw

\section{Abstract}

\section{Background}

24 This study assessed whether serum lipid levels are associated with the risk of symptomatic

25 intracerebral hemorrhage ( $\mathrm{sCH})$ and functional outcomes in patients with acute ischemic stroke

26 after receiving intravenous thrombolysis.

\section{Methods}

28 We retrospectively analyzed consecutive ischemic stroke patients who were treated with

29 intravenous tissue plasminogen activator between January 2007 and January 2017. Lipid levels

30 on admission, including total cholesterol, low-density lipoprotein cholesterol (LDL-C), high-

31 density lipoprotein cholesterol (HDL-C), and triglyceride levels, as well as potential predictors of

32 sICH were tested using univariate and multivariate analyses.

\section{Results}

34 Of the 229 enrolled patients (100 women, aged $68 \pm 13$ years), 14 developed sICH and 103

$35(45 \%)$ had favorable functional outcomes at 3 months. The patients with sICH more often had

36 diabetes mellitus ( $71 \%$ vs. $26 \%, \mathrm{P}=0.01)$ and had more severe stroke (mean National Institutes

37 of Health Stroke Scale [NIHSS] score of 16 vs. 13, P = 0.045). Regarding lipid subtype, total

38 cholesterol, LDL-C, HDL-C, and triglyceride levels were not associated with sICH or functional 
39 outcomes. According to the results of multivariate analysis, the frequency of sICH was

40 independently associated with diabetes mellitus (odds ratio $[\mathrm{OR}]=6.04 ; 95 \%$ confidence

41 interval $[\mathrm{CI}]=1.31-27.95 ; \mathrm{P}=0.02)$ and the NIHSS score $(\mathrm{OR}=1.12 ; 95 \% \mathrm{CI}=1.02-1.22 ; \mathrm{P}=$

42 0.01). A higher NIHSS score was independently associated with unfavorable functional

43 outcomes $(\mathrm{OR}=0.86 ; 95 \% \mathrm{CI}=0.81-0.91 ; \mathrm{P}<0.001)$.

\section{Conclusions}

45 Serum lipid levels on admission, including total cholesterol, LDL-C, HDL-C, and triglyceride

46 levels, were not associated with sICH or 3-month functional outcomes after intravenous

47 thrombolysis for acute ischemic stroke.

48

49

50

51

52

53

54

55

56 
58 Introduction

59 Stroke is the second leading cause of death worldwide, and the overall global burden of stroke is

60 high and still increasing (Feigin et al., 2014). Several predictors of ischemic stroke have been

61 examined, including age, diabetes mellitus, cardiac disease, hypertension, cardioembolic

62 etiology, and dyslipidemia. The standard of care for acute ischemic stroke comprises

63 endovascular treatment and intravenous thrombolysis (Bhaskar et al., 2018). Intravenous

64 thrombolysis with recombinant tissue plasminogen activator (rt-PA) given within 4.5 hours of

65 stroke onset is effective in the acute stage of ischemic stroke (Hacke et al., 2008). However, the

66 intravenous administration of rt-PA induces a 10-fold increase in symptomatic intracerebral

67 hemorrhage (sICH) of brain infarction through breakdown of the neurovascular unit (Suzuki et

68 al., 2016). Post-thrombolytic sICH resulted in poor outcomes, with the mortality rate reaching

$698 \%$ at 3 months (Strbian et al., 2011). Thus, identifying the risk of sICH in patients receiving

70 recanalization therapy is crucial. Epidemiology studies have suggested an association of low

71 total cholesterol and low-density lipoprotein cholesterol (LDL-C) levels with the risk of primary

72 intracerebral hemorrhage (ICH) (Amarenco et al., 2006; Sturgeon et al., 2007). However, the

73 association between serum lipid levels, which comprises total cholesterol, LDL-C, high-density 
74 lipoprotein cholesterol (HDL-C), and triglyceride levels, and the occurrence of post-thrombolytic

75 sICH remains controversial (Bang et al., 2007; Makihara et al., 2012; Messe et al., 2013; Rocco

76 et al., 2012; Uyttenboogaart et al., 2008). Many studies have suggested that Asians have a two

77 times higher risk of ICH than non-Asians. Thus, identifying the risk of sICH following

78 thrombolysis therapy is important in the Asian population (Shen et al., 2007; van Asch et al.,

79 2010).

80 The present study investigated whether serum lipid levels are associated with sICH occurrence and

81 functional outcomes in patients with acute ischemic stroke who received intravenous

82 administration of rt-PA. In addition, we tested the hypothesis that serum lipid levels are associated

83 with sICH after the intravenous administration of rt-PA for acute ischemic stroke.

84

85 Method

86 Patient selection

87 We retrospectively analyzed consecutive ischemic stroke patients who received intravenous

88 administration of rt-PA within 24 hours of stroke onset at three hospitals affiliated with the

89 Taipei Medical University System in Taiwan between January 2007 and January 2017. This

90 study was approved and the requirement to obtain a signed consent form for all patients was 
91 waived by the Institutional Review Board of Taipei Medical University (N201512020). All

92 patients underwent the standard treatment within 3 hours of stroke onset. Inclusion and exclusion

93 criteria for intravenous rt-PA treatment were mainly adopted from the National Institute of

94 Neurological Disorders and Stroke protocol and the protocol of the Taiwan Guidelines for the

95 Management of Stroke. Initial stroke severity was assessed using the National Institutes of

96 Health Stroke Scale (NIHSS). The levels of lipid subtypes, namely total cholesterol, HDL-C,

97 LDL-C, and triglycerides, were determined in the fasting state in morning following admission

98 to prevent the effect of meals on serum lipid levels. Data on demographics, vascular risk factors,

99 blood pressure, time from stroke onset until treatment, hemoglobin A1c level, and serum glucose

100 level on admission (nonfasting, principally obtained during the first assessment in the emergency

101 room) were recorded. We aimed to determine the relationship between serum glucose levels on

102 acute admission and stroke outcomes. The level of hemoglobin A1c and a history of diabetes

103 mellitus were used as surrogates for the baseline serum glucose level.

104

105 Outcome measures

106 Patients who received treatment were initially administered an intravenous rt-PA dose of 0.6-0.9

$107 \mathrm{mg} / \mathrm{kg}$ and then admitted to the neurology intensive care unit for observation according to the 
108 protocol of the Taiwan Guidelines for the Management of Stroke. sICH was considered a

109 neurological deterioration, which was defined as an increase in the NIHSS score of $>4$ points

110 within 36 hours following rt-PA treatment, along with the observation of a hematoma on a

111 noncontrast computed tomography scan of the brain (Hacke et al., 1998). Functional outcomes at

1123 months were assessed using the modified Rankin scale (mRS).

113

114 Statistical analysis

115 For continuous variables, Fisher's exact test was performed. For continuous variables,

116 independent samples Student's $t$ test or the Mann-Whitney U test was used after testing for

117 normality. Previous studies of patients who received intravenous rt-PA for stroke have reported

118 multiple clinical and demographic predictors of sICH (Lou et al., 2008). We performed an

119 univariate logistic regression analysis to predict the effect of the following potential predictors

120 on sICH following rt-PA: age, NIHSS score, sex, time until rt-PA treatment, laboratory

121 variables, vascular risk factors, and lipid subtype levels. The independent effect of lipid subtype

122 levels on sICH was investigated using a multivariable logistic regression model, in which the

123 potential predictors of sICH, namely the NIHSS score, serum glucose level, history of diabetes

124 mellitus, and lipid subtype levels, were tested. Given that the total cholesterol level was 
125 correlated with lipid subtype levels, we tested each lipid subtype separately in the regression

126 model. Finally, we used a multivariable regression analysis model to examine the relationship

127 between lipid levels and functional outcomes at 3 months. In this model, the following covariates

128 were entered: age, NIHSS score, serum glucose level, history of diabetes mellitus, and lipid

129 subtype levels. Variables were excluded if possible collinearity occurred (defined as Variance

130 Inflation Factor $>10$ ). Significance was defined as a $P$ value of $<0.05$. All statistical analyses

131 were performed using SPSS software, version 18.0 for Windows (SPSS, Chicago, Illinois).

132

133 Results

134 A total of 229 patients were included in this study. Their baseline demographic characteristics

135 are listed in Table 1. Of the 229 patients, sICH occurred in 14 (6.1\%) patients (95\% confidence

136 interval $[\mathrm{CI}]=3.0-9.0$ ). All the variables did not follow a normal distribution, except for the

137 mean systolic blood pressure. The median NIHSS score and a history of diabetes mellitus were

138 significantly associated with the risk of sICH. No difference was observed in the occurrence

139 sICH according to LDL, HDL, total cholesterol, and triglyceride levels. The comparison of

140 clinical and laboratory results between patients with and without sICH is shown in Table 2.

141 According to the results of the univariate analysis, the serum glucose level on admission (odds 
142 ratio $[\mathrm{OR}]=1.01 ; 95 \% \mathrm{CI}=1.00-1.01 ; \mathrm{P}=0.03)$ and a history of diabetes mellitus $(\mathrm{OR}=7.00$;

$14395 \% \mathrm{CI}=2.11-23.25 ; \mathrm{P}=0.001)$ were significantly associated with the occurrence of sICH

144 (Table 2). The total cholesterol, HDL-C and LDL-C levels appeared to be associated with lower

145 risk of sICH, although not significant $(\mathrm{OR}=0.995,0.997,0.98$, all P's $>0.05)$. After adjustment

146 for covariates in the multivariable regression model, the NIHSS score $(\mathrm{OR}=1.12 ; 95 \% \mathrm{CI}=$

$1471.02-1.22 ; \mathrm{P}=0.01)$ and a history of diabetes mellitus $(\mathrm{OR}=6.04 ; \mathrm{CI}=1.31-27.95 ; \mathrm{P}=0.02)$

148 were independently and significantly associated with sICH (Table 3). No significant association

149 was observed between lipid subtypes and sICH in the final multivariable regression model.

150 According to the results of the functional outcome analysis, at 3 months, all the patients with

151 sICH $(n=14)$ had unfavorable functional outcomes (mRS $\geq 3)$. Overall, 103 patients $(45 \%)$ had

152 favorable outcomes $(\mathrm{mRS} \leq 2)$ at 3 months (Table 1). Lipid subtype levels did not significantly

153 differ between the patients with favorable outcomes and those with unfavorable outcomes. After

154 adjustment for covariates in the multivariate analysis, a higher NIHSS score was found to be

155 independently associated with unfavorable outcomes $(\mathrm{OR}=0.86 ; \mathrm{CI}=0.81-0.91 ; \mathrm{P}<0.001)$.

156

157 Discussion 
158 The present study investigated the effects of lipid subtype levels on clinical outcomes in patients

159 with ischemic stroke following the intravenous administration of rt-PA. The major finding of this

160 study was that the levels of lipid subtypes, namely total cholesterol, LDL-C, HDL-C, and

161 triglycerides, were not associated with the occurrence of sICH or unfavorable outcomes

162 following rt-PA treatment. The NIHSS score and a history of diabetes mellitus were

163 independently and significantly associated with the risk of sICH. A higher NIHSS score

164 increased the odds for unfavorable outcomes at 3 months.

165 Our findings are in contrast to those of a previous study that reported an association between low

166 LDL-C levels and sICH (Bang et al., 2007). This discrepancy in findings may partly be

167 explained by differences in the study design. All our patients had received intravenous rt-PA.

168 However, Bang et al. investigated 104 patients who received thrombolysis with intravenous or

169 intra-arterial rt-PA and mechanical recanalization. In their study, only three (12\%) patients

170 developed sICH following the intravenous administration of rt-PA and the occurrence of sICH

171 was higher in patients (18\%) who received intra-arterial rt-PA and mechanical recanalization

$172(18 \%)$. Another two studies have identified triglycerides as an independent predictor of sICH in

173 patients with acute ischemic stroke (Messe et al., 2013; Uyttenboogaart et al., 2008).

174 Uyttenboogaart et al. analyzed 252 patients, all of whom received intravenous rt-PA, and found 
175 that a higher NIHSS score, prior antiplatelet use, and a higher triglyceride level were

176 independently associated with sICH. By contrast, Messe et al. included 22216 patients who

177 received intravenous rt-PA and demonstrated that higher HDL-C and lower triglyceride levels

178 were associated with a moderately increased risk of sICH. The inconsistent association between

179 triglycerides and sICH might be due to the measurement of lipids in different states in these

180 studies. In the study of Uyttenboogaart et al., serum lipid levels were tested in an acute phase and

181 nonfasting state. However, information regarding the time when lipid measurements were

182 performed was not provided in the study conducted by Messe et al. In our study, we measured

183 fasting lipid levels after the occurrence of acute ischemic stroke. The reason for this discrepancy

184 in triglyceride levels is not clear; however, acute psychological stress was reported to delay

185 serum triglyceride clearance in healthy, middle-aged men and women (Stoney et al. 2002).

186

187 Our results are compatible with those of the majority of studies that reported that the serum total

188 cholesterol or LDL-C level did not cause an increase in the risk of sICH or poor functional

189 outcomes in acute ischemic stroke following the intravenous administration of rt-PA (Makihara

190 et al., 2012; Nardi et al., 2012; Rocco et al., 2012). Consistent with our results, Makihara et al.

191 investigated 489 patients with acute stroke who received intravenous rt-PA and reported that no 
192 relationship existed between any lipid subtype level obtained in the acute phase and sICH,

193 whereas a higher HDL-C level was associated with favorable functional outcomes at 3 months.

194 In addition, Nardi et al. included a large cohort of 1847 patients with acute stroke; they found no

195 association between lipid levels and sICH; however, they reported that low HDL-C and

196 triglyceride levels were independently associated with mortality. Rocco et al. investigated 1066

197 patients and reported no association between the fasting lipid level in the acute phase and sICH

198 in patients treated with rt-PA. The same result was found for mortality or functional outcomes at

1993 months. Likewise, our data showed that lipid levels were not related to unfavorable outcomes.

200 A rapid decrease in serum lipid levels is observed in acute illness and is considered to be

201 connected with active inflammation (Esteve et al., 2005). Most prior studies that evaluated the

202 association between thrombolysis and sICH have reported results based on serum lipid levels

203 measured at a single time point. However, cholesterol and triglyceride levels are not reliable

204 when not measured in a fasting state and may decrease in the acute phase of stroke (Phuah et al.,

205 2016). Furthermore, a temporal association of a decline in serum lipid levels, which was a proxy

206 for increasing systemic inflammation, within 6 months preceding the occurrence of ICH was

207 found in a previous study (Phuah et al., 2016). Thus, the discrepancy in the results of prior

208 studies may partly be due to the timing of serum lipid level measurements. 
209 The mechanism underlying the relationship between lipid levels and ICH remains unclear. A

210 meta-analysis that included approximately 800 patients with acute ischemic stroke, most of

211 whom were not treated with intravenous rt-PA, showed that ICH tended to occur more often in

212 patients with lower LDL-C levels, whereas HDL-C and triglyceride levels were not associated

213 with the occurrence of ICH (Nardi et al., 2011). By contrast, a recent genetic study found that

214 high serum total cholesterol and LDL-C levels increased the risk of ICH, thereby providing a

215 more reliable lifetime exposure risk (Akoudad et al., 2016). In our study, lipid levels were

216 measured only once in the fasting state in the acute phase of stroke. Therefore, we do not have an

217 accurate explanation for how temporal lipid levels affect the occurrence of sICH following

218 thrombolysis. We speculate that serum lipid levels measured in the acute phase of stroke is not

219 an independent risk factor for sICH after thrombolysis in the acute phase of stroke. The results of

220 our study, compatible with the two major risk factors in HAT score, showed that a history of

221 diabetes mellitus and a higher NIHSS score were independently associated with the incidence of

222 sICH after thrombolysis (Lou et al.. 2008; Messe et al., 2013). Furthermore, a history of diabetes

223 mellitus was not associated with unfavorable outcomes at 3 months. This result supports the

224 finding that a history of diabetes mellitus is not a contraindication to intravenous adminsitration

225 of rt-PA (Mishra et al., 2011; Reiter et al., 2014). 
227 This study has several limitations. This retrospective, observational, case-control study included

228 only post-thrombolytic patients from three hospitals located in Northern Taiwan; this may have

229 introduced selection bias and diminished the generalizability of our results. The sample size of

230 patients who had sICH and poor functional outcomes was small, which limits the use of

231 regression models for the outcome analysis. Moreover, other potential risk factors for sICH, such

232 as early signs of ischemia on brain imaging, recanalization status, and previous statin use, were

233 not consistently evaluated for the entire cohort. However, many studies have reported that

234 previous statin use was not independently associated with $\mathrm{sICH}$ following rt-PA treatment

235 (Makihara et al., 2012; Messe et al., 2013; Nardi et al., 2012; Rocco et al., 2012; Uyttenboogaart

236 et al., 2008). Because of the fact that serum lipid levels were measured only once, the association

237 may be explained by remaining confounding factors due to the presence of undetected factors

238 such as diet (Durrington 1990). Additionally, information on the body mass index, which was

239 shown to inversely increase the risk of primary ICH, was missing in our study (Lioutas et al.,

240 2017). Finally, unmeasured confounding socioeconomic variables, such as the educational status,

241 may have influenced the risk of stroke (Jackson, 2018). 
242 In summary, total cholesterol, LDL-C, HDL-C and triglyceride levels were not associated with

243 sICH or unfavorable functional outcomes after thrombolysis therapy in acute ischemic stroke.

244 The clinical application requires further research to confirm these results.

245

246 Funding/Support Statement : This study was supported by Taipei Medical University Hospital

247 (104TMUH-NE-01). There was no additional external funding received for this study.

248 Acknowledgements: This manuscript was edited by Wallace Academic Editing.

249

250

251

252

253

254

255

256

257

258

259

260

261

262

263

264

265

266

267

268

\section{References}

Akoudad S, Ikram MA, Portegies ML, Adams HH, Bos D, Hofman A, Koudstaal PJ, Uitterlinden AG, van der Lugt A, van Duijn CM, and Vernooij MW. 2016. Genetic loci for serum lipid fractions and intracerebral hemorrhage. Atherosclerosis 246:287-292. 10.1016/j.atherosclerosis.2016.01.024

Amarenco P, Bogousslavsky J, Callahan A, 3rd, Goldstein LB, Hennerici M, Rudolph AE, Sillesen H, Simunovic L, Szarek M, Welch KM, Zivin JA, and Stroke Prevention by Aggressive Reduction in Cholesterol Levels I. 2006. High-dose atorvastatin after stroke or transient ischemic attack. N Engl J Med 355:549-559. 10.1056/NEJMoa061894

Bang OY, Saver JL, Liebeskind DS, Starkman S, Villablanca P, Salamon N, Buck B, Ali L, Restrepo L, Vinuela F, Duckwiler G, Jahan R, Razinia T, and Ovbiagele B. 2007. Cholesterol level and symptomatic hemorrhagic transformation after ischemic stroke thrombolysis. Neurology 68:737-742. 10.1212/01.wnl.0000252799.64165.d5

Bhaskar S, Stanwell P, Cordato D, Attia J, and Levi C. 2018. Reperfusion therapy in acute ischemic stroke: dawn of a new era? BMC Neurol 18. 10.1186/s12883-017-1007-y

Durrington PN. 1990. Biological variation in serum lipid concentrations. Scand J Clin Lab Invest Suppl 198:86-91.

Esteve E, Ricart W, and Fernandez-Real JM. 2005. Dyslipidemia and inflammation: an evolutionary conserved mechanism. Clin Nutr 24:16-31. 10.1016/j.clnu.2004.08.004 
269

270

271

272

273

274

275

276

277

278

279

280

281

282

283

284

285

286

287

288

289

290

291

292

293

294

295

296

297

298

299

300

301

302

303

304

Feigin VL, Forouzanfar MH, Krishnamurthi R, Mensah GA, Connor M, Bennett DA, Moran AE, Sacco RL, Anderson L, Truelsen T, O'Donnell M, Venketasubramanian N, Barker-Collo S, Lawes CM, Wang W, Shinohara Y, Witt E, Ezzati M, Naghavi M, Murray C, Global Burden of Diseases I, Risk Factors S, and the GBDSEG. 2014. Global and regional burden of stroke during 1990-2010: findings from the Global Burden of Disease Study 2010. Lancet 383:245-254.

Hacke W, Kaste M, Bluhmki E, Brozman M, Davalos A, Guidetti D, Larrue V, Lees KR, Medeghri Z, Machnig T, Schneider D, von Kummer R, Wahlgren N, Toni D, and Investigators E. 2008. Thrombolysis with alteplase 3 to 4.5 hours after acute ischemic stroke. N Engl J Med 359:1317-1329. 10.1056/NEJMoa0804656

Hacke W, Kaste M, Fieschi C, von Kummer R, Davalos A, Meier D, Larrue V, Bluhmki E, Davis S, Donnan G, Schneider D, Diez-Tejedor E, and Trouillas P. 1998. Randomised double-blind placebo-controlled trial of thrombolytic therapy with intravenous alteplase in acute ischaemic stroke (ECASS II). The Lancet 352:1245-1251. 10.1016/s01406736(98)08020-9

Jackson CA. 2018. Education, sex and risk of stroke: a prospective cohort study in New South Wales, Australia. 8. 10.1136/bmjopen-2018-024070

Lioutas VA, Beiser A, Himali J, Aparicio H, Romero JR, DeCarli C, and Seshadri S. 2017. Lacunar infarcts and deep intracerebral hemorrhage differences: A nested case-control analysis in the Framingham Heart Study. Stroke 48:486-489. 10.1161/strokeaha.116.014839

Lou M, Safdar A, Mehdiratta M, Kumar S, Schlaug G, Caplan L, Searls D, and Selim M. 2008. The HAT Score: a simple grading scale for predicting hemorrhage after thrombolysis. Neurology 71:1417-1423. 10.1212/01.wnl.0000330297.58334.dd

Makihara N, Okada Y, Koga M, Shiokawa Y, Nakagawara J, Furui E, Kimura K, Yamagami H, Hasegawa Y, Kario K, Okuda S, Naganuma M, and Toyoda K. 2012. Effect of serum lipid levels on stroke outcome after rt-PA therapy: SAMURAI rt-PA registry. Cerebrovasc Dis 33:240-247. 10.1159/000334664

Messe SR, Pervez MA, Smith EE, Siddique KA, Hellkamp AS, Saver JL, Bhatt DL, Fonarow GC, Peterson ED, and Schwamm LH. 2013. Lipid profile, lipid-lowering medications, and intracerebral hemorrhage after tPA in get with the guidelines-stroke. Stroke 44:13541359. 10.1161/STROKEAHA.111.671966

Mishra NK, Ahmed N, Davalos A, Iversen HK, Melo T, Soinne L, Wahlgren N, Lees KR, Sits, and collaborators V. 2011. Thrombolysis outcomes in acute ischemic stroke patients with prior stroke and diabetes mellitus. Neurology 77:1866-1872.

10.1212/WNL.0b013e318238ee42

Peer] reviewing PDF | (2018:08:30495:1:0:NEW 17 Oct 2018) 
305

306

307

308

309

310

311

312

313

314

315

316

317

318

319

320

321

322

323

324

325

326

327

328

329

330

331

332

333

334

335

336

337

338

339

340

Nardi K, Engelter S, Strbian D, Sarikaya H, Arnold M, Casoni F, Ford GA, Cordonnier C, Lyrer P, Bordet R, Soinne L, Gensicke H, Duriez P, Baumgartner RW, Tatlisumak T, Leys D, and Lipid Profile in Thrombolysis Study G. 2012. Lipid profiles and outcome in patients treated by intravenous thrombolysis for cerebral ischemia. Neurology 79:1101-1108. 10.1212/WNL.0b013e3182608c82

Nardi K, Leys D, Eusebi P, Cordonnier C, Gautier S, Hénon H, and Bordet R. 2011. Influence of Lipid Profiles on the Risk of Hemorrhagic Transformation after Ischemic Stroke: Systematic Review. Cerebrovasc Dis Extra 1:130-141. 10.1159/000335014

Phuah CL, Raffeld MR, Ayres AM, Viswanathan A, Greenberg SM, Biffi A, Rosand J, and Anderson CD. 2016. Subacute decline in serum lipids precedes the occurrence of primary intracerebral hemorrhage. Neurology 86:2034-2041. 10.1212/WNL.0000000000002716

Reiter M, Teuschl Y, Matz K, Seyfang L, Brainin M, and Austrian Stroke Unit Registry C. 2014. Diabetes and thrombolysis for acute stroke: a clear benefit for diabetics. Eur J Neurol 21:5-10. 10.1111/ene.12263

Rocco A, Sykora M, Ringleb P, and Diedler J. 2012. Impact of statin use and lipid profile on symptomatic intracerebral haemorrhage, outcome and mortality after intravenous thrombolysis in acute stroke. Cerebrovasc Dis 33:362-368. 10.1159/000335840

Shen AY, Yao JF, Brar SS, Jorgensen MB, and Chen W. 2007. Racial/ethnic differences in the risk of intracranial hemorrhage among patients with atrial fibrillation. $J$ Am Coll Cardiol 50:309-315. 10.1016/j.jacc.2007.01.098

Stoney CM, West SG, Hughes JW, Lentino LM, Finney ML, Falko J, and Bausserman L. 2002. Acute psychological stress reduces plasma triglyceride clearance. Psychophysiology 39:80-85. 10.1017/s0048577202010284

Strbian D, Sairanen T, Meretoja A, Pitkaniemi J, Putaala J, Salonen O, Silvennoinen H, Kaste M, Tatlisumak T, and Helsinki Stroke Thrombolysis Registry G. 2011. Patient outcomes from symptomatic intracerebral hemorrhage after stroke thrombolysis. Neurology 77:341-348. 10.1212/WNL.0b013e3182267b8c

Sturgeon JD, Folsom AR, Longstreth WT, Jr., Shahar E, Rosamond WD, and Cushman M. 2007. Risk factors for intracerebral hemorrhage in a pooled prospective study. Stroke 38:27182725. 10.1161/STROKEAHA.107.487090

Suzuki Y, Nagai N, and Umemura K. 2016. A Review of the Mechanisms of Blood-Brain Barrier Permeability by Tissue-Type Plasminogen Activator Treatment for Cerebral Ischemia. Front Cell Neurosci 10:2. 10.3389/fncel.2016.00002

Uyttenboogaart M, Koch MW, Koopman K, Vroomen PC, Luijckx GJ, and De Keyser J. 2008. Lipid profile, statin use, and outcome after intravenous thrombolysis for acute ischaemic stroke. J Neurol 255:875-880. 10.1007/s00415-008-0797-7 
341 van Asch CJJ, Luitse MJA, Rinkel GJE, van der Tweel I, Algra A, and Klijn CJM. 2010.

342 Incidence, case fatality, and functional outcome of intracerebral haemorrhage over time, 343 according to age, sex, and ethnic origin: a systematic review and meta-analysis. The

344 Lancet Neurology 9:167-176. 10.1016/s1474-4422(09)70340-0

345 


\section{Table $\mathbf{1}$ (on next page)}

Baseline characteristics

HDL-Chigh-density lipoprotein cholesterol; IQRinterquartile range; LDL-Clow-density lipoprotein cholesterol; mRSmodified Rankin scale; NIHSSNational Institutes of Health Stroke Scale;rt-PArecombinant tissue plasminogen activator; sICHsymptomatic intracerebral hemorrhage; SDstandard deviation. 
1 Table 1 Baseline characteristics

2

3

4 Mean age (SD), years

5 Male, $\mathrm{n}(\%)$

6 Median NIHSS score [IQR]

7 Median time until treatment [IQR], min

8 Mean systolic blood pressure (SD), $\mathrm{mmHg}$

9 Mean diastolic blood pressure (SD), $\mathrm{mmHg}$

10 Mean admission serum glucose level (SD), mg/dL

11 Mean total cholesterol level (SD), mg/dL

12 Mean HDL-C level (SD), mg/dL

13 Mean LDL-C level (SD), mg/dL

14 Mean triglyceride level (SD), mg/dL

15 Mean prothrombin time-INR level, (SD)

16 Mean creatinine level (SD), mg/dL

17 Mean hemoglobin A1c level (SD), \%

18 Vascular risk factors

$\begin{array}{ll}19 & \text { History of arterial hypertension, n (\%) } \\ 20 & \text { Diabetes mellitus, n (\%) } \\ 21 & \text { Hyperlipidemia, n (\%) } \\ 22 & \text { Atrial fibrillation, n (\%) } \\ 23 & \text { Coronary heart disease, n (\%) } \\ 24 & \text { Current smoker, n (\%) } \\ 25 & \text { Previous stroke, n (\%) } \\ 26 & \text { Mean rt-PA dose } \\ 27 & \text { Favorable outcome (mRS } \leq 2)\end{array}$

\section{Total sICH no sICH P value}

$\mathrm{N}=229 \quad \mathrm{~N}=14 \quad \mathrm{~N}=215$

$\begin{array}{llll}68(13) & 72(10) & 67(13) & 0.20 \\ 129(56) & 8(57) & 121(56) & 0.95 \\ 13[8-18] & 16[13-22] & 13[8-18] & 0.045\end{array}$

$105[75-145] 120[83-152] 105[74-145] \quad 0.41$

$168(27) \quad 175(26) \quad 167(27) \quad 0.29$

$95(20) \quad 88(19) \quad 95(20) \quad 0.37$

$\begin{array}{llll}152(69) & 192(93) & 149(66) & 0.08\end{array}$

$184(41) \quad 176(40) \quad 185(41) \quad 0.75$

$45(13) \quad 44(18) \quad 45(12) \quad 0.84$

$118(34) \quad 102(29) \quad 119(34) \quad 0.12$

$125(77) \quad 135(85) \quad 125(77) \quad 0.76$

$1.08(0.72) \quad 1.02(0.08) \quad 1.09(0.75) \quad 0.54$

$1.07(0.43) \quad 1.13(0.53) \quad 1.06(0.42) \quad 0.74$

$\begin{array}{llll}6.60(1.56) & 7.46(2.25) & 6.46(1.39) & 0.28\end{array}$

$\begin{array}{llll}178(78) & 12(86) & 166(77) & 0.74 \\ 66(29) & 10(71) & 56(26) & 0.01 \\ 84(37) & 4(29) & 80(37) & 0.51 \\ 95(42) & 7(50) & 88(41) & 0.51 \\ 41(20) & 3(21) & 38(18) & 0.96 \\ 38(21) & 1(7) & 37(17) & 0.46 \\ 25(11) & 1(7) & 24(11) & 1.00 \\ 0.83(0.13) & 0.86(0.09) & 0.83(0.13) & 0.59 \\ 103(45) & 0(0) & 103(48) & <0.000\end{array}$

$28 H D L-C$ high-density lipoprotein cholesterol; IQR interquartile range; $L D L-C$ low-density lipoprotein cholesterol;

$29 m R S$ modified Rankin scale; NIHSS National Institutes of Health Stroke Scale; $r t-P A$ recombinant tissue

30 plasminogen activator; sICH symptomatic intracerebral hemorrhage; SD standard deviation. 


\section{Table 2 (on next page)}

Univariate and multivariable regression analysis regarding the occurrence of $\mathrm{sICH}$

HDL-Chigh-density lipoprotein cholesterol; LDL-Clow-density lipoprotein cholesterol;

NIHSSNational Institutes of Health Stroke Scale; sICHsymptomatic intracerebral hemorrhage. 
1 Table 2 Univariate and multivariable regression analysis regarding the occurrence of sICH 2

3 3

5 Male

6 NIHSS, per point increase

Univariate regression analysis

Multivariable regression analysis

7 Time until treatment OR $\quad 95 \% \mathrm{CI} \quad \mathrm{P}$ value

OR

$95 \% \mathrm{CI} \quad \mathrm{P}$ value

8 Systolic blood pressure

1.03

$0.98-1.08$

0.21

1.04

0.35-3.09 0.95

$1.08 \quad 1.00-1.16 \quad 0.05$

1.12

$1.02-1.22 \quad 0.01$

9 Diastolic blood pressure

1.001

$0.99-1.01 \quad 0.78$

1.01

$0.99-1.03 \quad 0.29$

10 Admission serum glucose level

0.98

0.93-1.04 0.46

11 Total cholesterol level

1.01

$1.00-1.01$

0.03

1.01

0.996-1.02 0.26

12 HDL-C level

0.995

0.98-1.01

0.46

0.997

0.95-1.04

0.90

0.98

0.97-1.00 $\quad 0.10$

1.003

0.96-1.05 0.90

13 LDL-C level

14 Triglyceride level

1.002

0.995-1.01 0.63

0.99

0.97-1.00 $\quad 0.10$

15 Prothrombin time-INR level

0.68

0.05-9.06 $\quad 0.77$

16 Creatinine

1.45

$0.4-5.22 \quad 0.58$

17 Hemoglobin A1c

1.42

$0.95-2.13 \quad 0.09$

18 History of arterial hypertension

1.74

$0.38-8.02 \quad 0.48$

19 Diabetes mellitus

7.00

2.11-23.25 0.001

6.04

$1.31-27.95 \quad 0.02$

20 Hyperlipidemia

0.67

$0.20-2.21 \quad 0.51$

21 Atrial fibrillation

1.43

$0.49-4.23 \quad 0.52$

22 Coronary heart disease

0.98

$0.70-1.36 \quad 0.88$

23 Current smoker

0.30

0.04-2.36 0.25

24 Previous stroke

0.60

$0.8-4.79 \quad 0.63$

$25 H D L-C$ high-density lipoprotein cholesterol; $L D L-C$ low-density lipoprotein cholesterol; NIHSS National Institutes

26 of Health Stroke Scale; sICH symptomatic intracerebral hemorrhage. 


\section{Table 3(on next page)}

Multivariable regression analysis: association of lipid levels with favorable outcomes

HDL-Chigh-density lipoprotein cholesterol; LDL-Clow-density lipoprotein cholesterol;

NIHSSNational Institutes of Health Stroke Scale. 
1 Table 3 Multivariable regression analysis: association of lipid levels with favorable outcomes

2

$\begin{array}{lllll}3 & \text { Age } & 0.99 & 0.96-1.02 & 0.40 \\ 4 & \text { NIHSS } & 0.86 & 0.81-0.91 & <0.001 \\ 5 & \text { Admission serum glucose level } & 0.997 & 0.99-1.00 & 0.38 \\ 6 & \text { History of diabetes mellitus } & 0.53 & 0.96-1.02 & 0.14 \\ 7 & \text { LDL-C level } & 1.002 & 0.99-1.01 & 0.67 \\ 8 & \text { HDL-C level } & 0.99 & 0.96-1.02 & 0.41 \\ 9 & \text { Triglyceride level } & 1.002 & 0.997-1.01 & 0.46\end{array}$

$10 H D L-C$ high-density lipoprotein cholesterol; $L D L-C$ low-density lipoprotein cholesterol; NIHSS National Institutes of Health Stroke Scale. 